\title{
Movement and Motion of Soybean Cyst Nematode Heterodera glycines Populations and Individuals in Response to Abamectin
}

\author{
Jared P. Jensen, Augustine Q. Beeman, Zach L. Njus, Upender Kalwa, Santosh Pandey, and Gregory L. Tylka
}

First, second, and sixth authors: Department of Plant Pathology and Microbiology, 351 Bessey Hall, and third, fourth, and fifth authors: Department of Electrical and Computer Engineering, 2215 Coover Hall, Iowa State University, Ames 50011. Current address of J. P. Jensen: AgBiome, Research Triangle Park, NC 27709. Accepted for publication 29 January 2018.

\begin{abstract}
Two new in vitro methods were developed to analyze plant-parasitic nematode behavior, at the population and the individual organism levels, through time-lapse image analysis. The first method employed a highresolution flatbed scanner to monitor the movement of a population of nematodes over a $24-\mathrm{h}$ period at $25^{\circ} \mathrm{C}$. The second method tracked multiple motion parameters of individual nematodes on a microscopic scale, using a high-speed camera. Changes in movement and motion of second-stage juveniles (J2) of the soybean cyst nematode Heterodera glycines Ichinohe were measured after exposure to a serial dilution of

abamectin $(0.1$ to $100 \mu \mathrm{g} / \mathrm{ml})$. Movement and motion of $H$. glycines were significantly reduced as the concentration of abamectin increased. The effective range of abamectin to inhibit movement and motion of $H$. glycines $\mathrm{J} 2$ was between 1.0 and $10 \mu \mathrm{g} / \mathrm{ml}$. Proof-of-concept experiments for both methods produced one of the first in vitro sensitivity studies of $H$. glycines to abamectin. The two methods developed allow for higher-throughput analysis of nematode movement and motion and provide objective and data-rich measurements that are difficult to achieve from conventional microscopic laboratory methods.
\end{abstract}

In the United States, the soybean cyst nematode Heterodera glycines Ichinohe is arguably the principal yield-reducing pathogen of soybean year after year (Allen et al. 2017; Koenning and Wrather 2010). Nematode-protectant seed treatments are an emerging tool for managing $H$. glycines. Seed treatments are an attractive management option because they deliver a concentrated active ingredient (i.e., nematicide) directly around the developing seedling while minimizing the overall amount of chemical input used in the field (Munkvold et al. 2014). Additionally, combining the use of nematodeprotectant seed treatments with $H$. glycines-resistant soybean cultivars may reduce selection pressure for increased nematode virulence and prolong the efficacy of $H$. glycines-resistant soybean varieties. The emergence of and interest in nematode-protectant seed treatments has renewed the need to develop newer nematicides and newer methods to screen and study the effects of such compounds.

Juvenile mortality, as indicated by lack of movement, is a major defining factor of nematicide activity. A common laboratory method used to assess nematode mortality is observation of nematode movement using a light microscope. Some nematodes such as H. glycines may be immobile when observed for a short period of time but still be alive (Schroeder and MacGuidwin 2010). To confirm nematode death during a single observation, the observer must visually score the nematode response after prodding with a fine needle probe (Cayrol et al. 1986), stain the nematode with a vital stain (Carins et al. 1960; Hooper 1986; Ogiga and Estey 1974; Schroeder and MacGuidwin 2010), or stimulate the nematode with a basic compound such as sodium hydroxide (Chen and Dickson

${ }^{\dagger}$ Corresponding author: G. L. Tylka. E-mail: gltylka@iastate.edu

Funding: This work was supported by the National Science Foundation I/UCRC, the Center for Arthropod Management Technologies under grant number IIP1338775; by industry partners; and by Syngenta, for providing financial support to J. P. Jensen.

*The $\boldsymbol{e}$-Xtra logo stands for "electronic extra" and indicates that one supplementary video is published online.

(C) 2018 The American Phytopathological Society
2000; Xiang and Lawrence 2016). These methods are effective but they subjectively measure only the presence or absence of movement during a single observation. Analysis of movement and motion with time-lapse imaging can serve as an alternative in vitro method to quantitatively study nematode death through the analysis of behavioral response to nematicides and also may allow detection of sublethal effects of compounds causing subtle changes in nematode behavior.

Sequential, digital imaging has been used to investigate nematode movement since the 1980 s. Several movement-analysis methods have been developed to study Caenorhabditis elegans and range from highthroughput analysis observing multiple nematodes to low-throughput, data-intensive motion analysis of individuals (Dusenbery 1985; Husson et al. 2005; Njus et al. 2015; Peet et al. 1990). These techniques, however, have not been used with plant-parasitic nematodes, some of which exhibit sedentary or slow-moving behavior (Husson et al. 2005). Recently, image analysis and nematode-tracking techniques have been adapted to be used for studies with plant-parasitic nematodes. For example, Beeman et al. (2016) used images obtained from a highresolution flatbed scanner to monitor nematode chemotaxis in response to live soybean seedling root exudates in a microfluidic chip.

The adaptation of time-lapse image analysis tools to study plantparasitic nematodes would provide researchers unbiased, highthroughput methods to aid in the development of new nematicides. Data-rich analyses of nematode behavior will enable the detection of both lethal and sublethal effects of nematicides, potentially resulting in identification of new nematicidal compounds with a wide range of modes of action. The objective of our work was to develop new image-analysis techniques to study nematode behavior through movement and motion analysis of $H$. glycines second-stage juveniles (J2). In this article, behavioral analysis of populations of $\mathrm{J} 2$ is referred to as "movement", whereas behavioral analysis of individual $\mathrm{J} 2$ is referred to as "motion".

\section{MATERIALS AND METHODS}

Nematode preparation. The $H$. glycines populations used in these experiments were maintained in greenhouse culture pots on H. glycines-susceptible soybean Glycine max 'Williams 82' grown 
in sandy soil that was naturally infested with $H$. glycines (HG type 2.5.7). H. glycines females were removed from 30 -day-old soybean roots by spraying the roots with a water stream, with the roots placed on a $850-\mu \mathrm{m}$-pore sieve nested over a $250-\mu \mathrm{m}$-pore sieve. The females collected on the lower, 250- $\mu \mathrm{m}$-pore sieve were separated from soil particles and other debris via sucrose centrifugation using a sucrose/ water solution at 1,362 g/liter (Jenkins 1964). Next, the females were crushed using a rotating rubber stopper against a $250-\mu \mathrm{m}$-pore sieve to release the eggs (Faghihi and Ferris 2000). The eggs were collected on a $25-\mu \mathrm{m}$-pore sieve nested under a $75-\mu \mathrm{m}$-pore sieve and were further isolated from debris through a second round of sucrose centrifugation with a sucrose/water solution at $454 \mathrm{~g} /$ liter (Jenkins 1964). The eggs

A
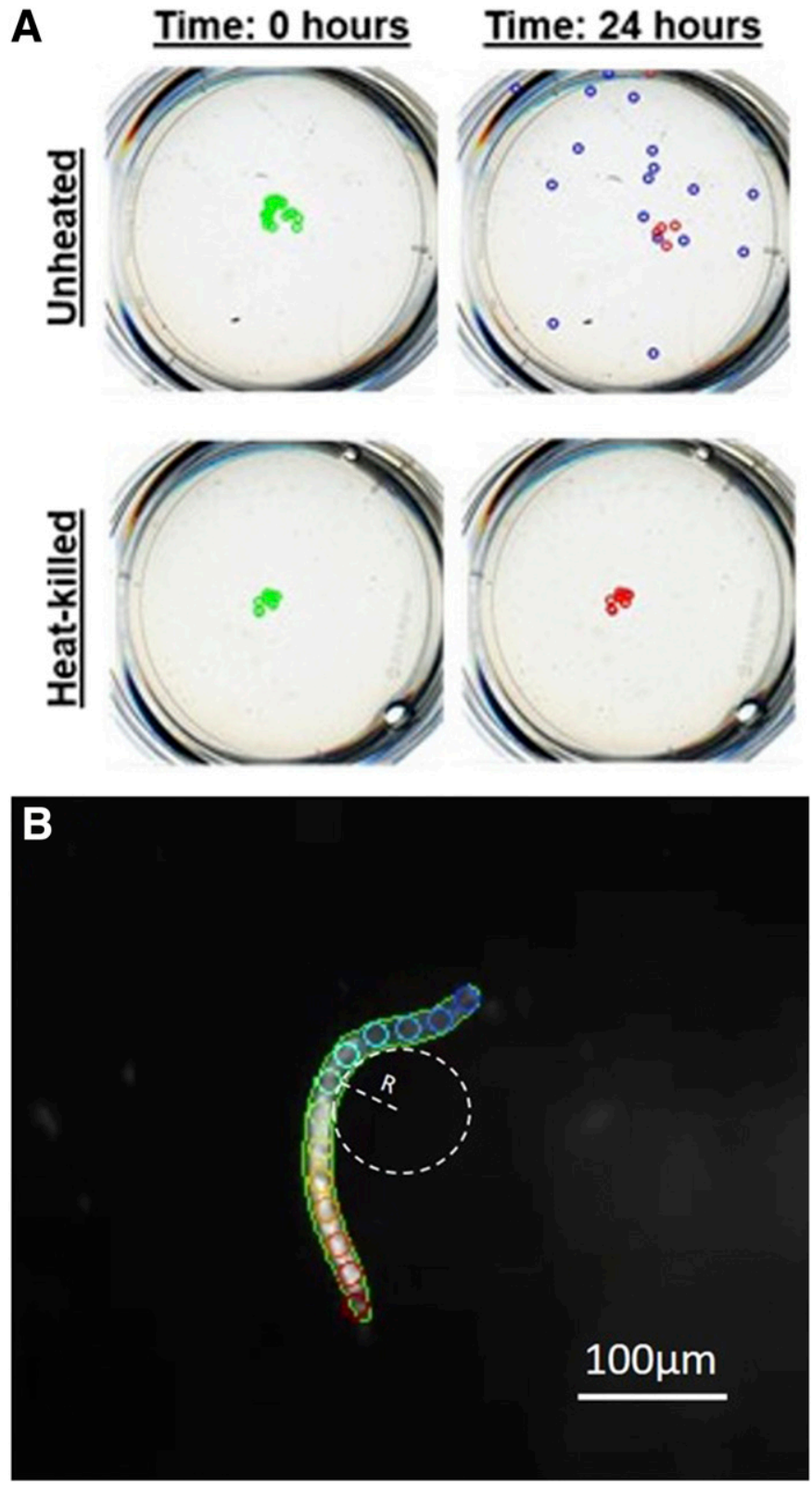

Fig. 1. Methods to measure nematode behavior in movement analysis of nematode populations and motion analysis of individual nematodes. A, Preliminary experiments analyzed movement of two nematode populations over every hour for $24 \mathrm{~h}$, with representative observations at hours 0 and 24. Circles represent individual nematodes. Blue circles indicate that nematode had moved since the previous observation. Red circles indicate that the nematode did not move since the previous observation. B, Diagram of 13 evenly spaced tracking points (multicolored circles) used for motion analysis of individual nematodes. Curvature was measured by calculating the reciprocal of the radius (R) of a circle that passed through three points: the tracking point of interest and its two adjacent points. were rinsed with water, then incubated on a $30-\mu \mathrm{m}$-pore sieve over a thin film of tap water at $25^{\circ} \mathrm{C}$ for $72 \mathrm{~h}$ to collect hatched $\mathrm{J} 2$ (Wong et al. 1993). To obtain active nematodes, collected $\mathbf{J} 2$ were once more placed on a $30-\mu \mathrm{m}$-pore sieve in $1 \mathrm{~cm}$ of tap water. After $1 \mathrm{~h}, \mathrm{~J} 2$ that moved through the sieve were collected in the water below and used for movement and motion analysis experiments.

Abamectin incubation. Reagent-grade abamectin $\mathrm{B}_{1}$ a (CAS: 71751-41-2, >97\% purity; Alfa Aesar, Haverhill, MA), a known nematicide (Kass et al. 1984; Wright et al. 1983, 1984), was dissolved in $100 \%$ acetone, then serially diluted to final concentrations of abamectin of $100,10,1$, and $0.1 \mu \mathrm{g} / \mathrm{ml}$ in $1 \%$ acetone. The $1 \%$ acetone solvent acted as a control treatment and is henceforth referred to as the abamectin concentration of $0 \mu \mathrm{g} / \mathrm{ml}$. Active populations of $H$. glycines $\mathrm{J} 2$, collected as described above, were incubated in one of the abamectin concentrations or in sterile distilled water in the dark at $25^{\circ} \mathrm{C}$ for $2 \mathrm{~h}$. Next, the incubated $\mathrm{J} 2$ were rinsed three times with sterile distilled water to remove excess abamectin. Heat-killed J2, boiled in water for $5 \mathrm{~min}$, were included as a negative control treatment. In summary, $H$. glycines $\mathrm{J} 2$ were incubated in one of five different concentrations of abamectin or in sterile water, or were heat killed. The movement and motion of $\mathrm{J} 2$ from each treatment were analyzed with the two methods described below.

Movement analysis of nematode populations. A population of 15 to $60 \mathrm{H}$. glycines $\mathrm{J} 2$ from each treatment was added to the center of individual wells of a 24-well plate containing $11.5 \%$ Pluronic F-127 gel (Sigma-Aldrich, St. Louis) (Sasaki-Crawley et al. 2012). The plates were placed onto an EPSON Perfection V750 Pro scanner, positioned inside a temperature-controlled plexiglass housing at $25^{\circ} \mathrm{C}$, where scanned images at 2,400 dpi were captured every hour for $24 \mathrm{~h}$. Using a custom program written in MATLAB (Mathworks Inc., Natick, MA), the researcher highlighted the location of every nematode within an observation or image. The program then assigned $\mathrm{X}$ and $\mathrm{Y}$ coordinates for each nematode in each image, and the coordinates of the positions of the nematodes were compared between images to determine the movement of the nematode population. It was not possible to follow the movements of individual nematodes over time because the software did not assign a unique identity to each nematode. The software only identified net differences in the positions of unidentified nematodes between images. Movement within the nematode population was considered to have occurre if an $\mathrm{X}$ and $\mathrm{Y}$ coordinate pair from one image was at least $300 \mu \mathrm{m}$ apart from what was observed in the subsequent image, because this distance was found to be an effective minimum threshold for movement in preliminary experiments (Fig. 1A). Using a minimum movement threshold of $300 \mu \mathrm{m}$ removed small artifacts in the data caused by displacement of heat-killed nematodes due to scanner vibrations, nematode settling, or other external factors. For each treatment, populations of 15 to 60 nematodes were analyzed for six replications over $24 \mathrm{~h}$ with two experimental runs.

Motion analysis of individual nematodes. Nematodes in $30-\mu l$ suspensions from each treatment were placed on a microscope slide with a square, 20-mm coverslip. In total, 10 to 5 individual J2 of each treatment were observed using bright-field microscopy with overhead lighting (Leica LED 5000 light attached to Leica M205 C stereoscope $)$ at $\times 63$ magnification $(\times 6.3$ zoom, $\times 10$ eyepiece, and $\times 1$ camera). Overhead light provided optimal contrast between the nematode body and background for computer analysis. The motion of each nematode was recorded for $1 \mathrm{~min}$ with a high-speed camera (QICAM 12-bit colorfast 1394) at a frame rate of one image every $0.1 \mathrm{~s}$ (Supplementary Video S1). Videos were captured at 0 and $24 \mathrm{~h}$ after incubation. The videos were analyzed with another custom program written in MATLAB that tracked 13 evenly spaced points along the nematode body (Fig. 1B). In the preliminary experiments, 13 tracking points were determined to be a sufficient number for $H$. glycines (data not shown); more tracking points are needed with longer nematodes such as Brugia malayi (Njus 2017). An X and Y coordinate was assigned for each tracking point at successive time 
points within the software, and these coordinates were used by the program to calculate speed and change in curvature of the nematodes. Speed, in micrometers per second, was calculated as the change in distance between $\mathrm{X}$ and $\mathrm{Y}$ coordinates of a single tracking point between successive images $(10 \mathrm{~ms})$. Curvature, in micrometers, was calculated using the Menger curvature method (Korta et al. 2007) in which curvature is represented as the radius ( $r$ ) of a circle that passes through three points, centered on the point of interest (Fig. 1B). The change in curvature was calculated as the change in radius length $\left(\mathrm{r}^{-1}\right)$ between successive time points. Given this formula, the anterior- and posteriormost tracking points on the nematode cannot be analyzed for curvature because there is not an adjacent point on either side of those two tracking points. Consequently, only 11 tracking points were used. Finally, the MATLAB program combined all the tracking points of an individual nematode to produce a contour plot, which illustrated the changes in nematode curvature over time. The motion of at least six individual J2 was analyzed per treatment, and the experiment was conducted twice.

Data analysis. All statistical analyses were completed using JMP Pro 13 (SAS Institute, Cary, NC). Analysis of variance $(P=$ $0.05)$ was used to determine significance of effects of factors for each assay. A repeated measures analysis was conducted using a mixed model to determine treatment effects for movement analysis when averaged over the entire $24 \mathrm{~h}$. When significant treatment effects were detected, means were separated using Tukey's honestly significance difference test $(\alpha=0.05)$. The means tested were abamectin concentration, time, body location (individual motion analysis only), and experimental run. For the motion analysis of individual nematodes, these effects were calculated for both movement parameters (speed and change in curvature).

\section{RESULTS}

Movement analysis of nematode populations. When averaged over $24 \mathrm{~h}$, the percent movement of heat-killed J2 was $1.6 \%$ compared with the $42.1 \%$ movement in water and $43.0 \%$ in abamectin at $0 \mu \mathrm{g} / \mathrm{ml}$ (data not shown). The highest concentration of abamectin $(100 \mu \mathrm{g} / \mathrm{ml})$ significantly reduced nematode movement to $1.9 \%$ of the population, an approximately $95 \%$ reduction compared with the observed movement of the population incubated in water. Abamectin concentrations of 1 and $10 \mu \mathrm{g} / \mathrm{ml}$ significantly reduced movement of the population, similar to the $100 \mu \mathrm{g} / \mathrm{ml}$ concentration. The lowest concentrations of abamectin $(0$ and $0.1 \mu \mathrm{g} / \mathrm{ml}$ ) did not reduce population movement relative to the water treatment. There was elevated movement of $\mathrm{J} 2$ in all treatments at the beginning of the experiment; then, movement became more stable over the final $10 \mathrm{~h}$ of analysis (Fig. 2).

Motion analysis of individual nematodes. The motion of individual $H$. glycines $\mathrm{J} 2$ was illustrated in contour plots with data from representative $\mathbf{J} 2$, selected arbitrarily, from each treatment (Fig. 3A to G). In these plots, the change in curvature was plotted for all 11 possible sets of tracking points over the 60-s observation period. An active worm had shorter wavelengths and higher frequencies of motion, as depicted by recurring vertical bands of shorter widths (e.g., in the control). In contrast, an inactive worm had longer wavelengths and lower frequencies or motion, represented by wider or nonchanging bands (as shown in higher abamectin concentrations). Higher numbers of vertical lines throughout a contour plot indicated more changes in curvature. In the lowest abamectin concentrations ( 0 and $0.1 \mu \mathrm{g} / \mathrm{ml}$ ), the nematodes exhibited multiple changes in curvature, similar to that of nematodes in the water control. The $\mathrm{J} 2$ incubated in the highest abamectin concentration $(100 \mu \mathrm{g} / \mathrm{ml})$ exhibited no change in curvature, similar to the heat-killed negative control.

The pattern of the contour plots revealed that most of the nematode motion occurred at the head and tail tracking points; therefore, these two points were further analyzed quantitatively. Average speed of motion and change in curvature were both affected by abamectin concentration and varied by body location, time of image capture, and experimental run (data not shown). The average speed of the head and tail tracking points for the heat-killed treatment was 0.3 and $0.2 \mu \mathrm{m} / \mathrm{s}$, respectively. The average speed of the water treatment was 4.8 and $3.2 \mu \mathrm{m} / \mathrm{s}$ for the head and tail, respectively. As the concentrations of abamectin increased to 10 and $100 \mu \mathrm{g} / \mathrm{ml}$, speed of nematode motion was reduced across both body locations at 0 - and 24-h observation times (Fig. 4A and B, respectively) to levels similar to that of the heat-killed control nematodes. Similar results were obtained with change in curvature data collected at both 0 and $24 \mathrm{~h}$ (Fig. 5A and B, respectively). The average change in curvature for the heat-killed treatment was $1.6 \times 10^{-4}$ and $1.5 \times 10^{-4} \mu \mathrm{m}^{-1}$ for the head and tail, respectively, whereas change in curvature at these points on the nematode body in the water treatment was $1.98 \times 10^{-3}$ and $7.6 \times 10^{-4} \mu \mathrm{m}^{-1}$ for the head and tail, respectively. The two lowest concentrations of abamectin $(0$ and $0.1 \mu \mathrm{g} / \mathrm{ml})$ did not affect nematode speed and curvature; motion of the head and tail was similar to the water control. Motion of the head tracking point was significantly different from the tail when measured for both speed and change in curvature across all treatments.

\section{DISCUSSION}

Determining the response of plant-parasitic nematodes to experimental compounds often is a time-intensive, low-throughput task. Assessing nematode mortality by assessing lack of movement through a microscope yields a single, binary "moving or not moving" observation. Also, it is difficult to distinguish subtle differences in nematode behavior from a single visual observation. Manual observation methods are labor intensive and can be prone to interoperator variability and error from operator fatigue, resulting in false-positive and false-negative assessments. Therefore, there is a need to develop computer-based nematode observation techniques to accurately assess and quantify the behavior of plant-parasitic nematodes. Observations of nematode behavior through time-lapse image analysis offers an alternative way to determine nematode death, defined by lack of movement. Two new methods were developed that objectively quantify plant-parasitic nematode behavior through movement analysis of nematode populations and motion analysis of individual nematodes.

Movement analysis of nematode populations measured the percent movement of a population, defined as the proportion of nematodes in a population that had different $\mathrm{X}$ and $\mathrm{Y}$ coordinates compared with a previous observation. The sensitivity of this technique was validated by comparing percent movement

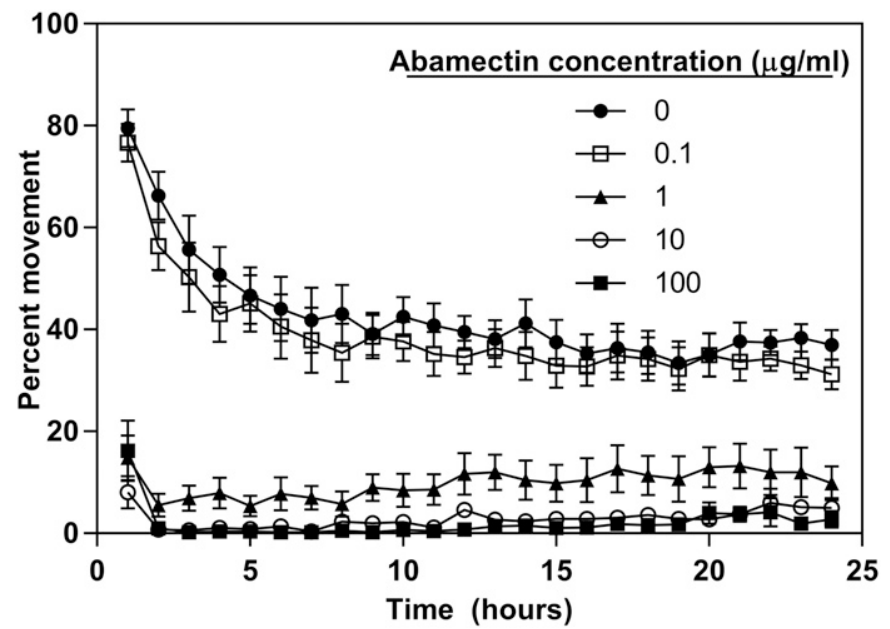

Fig. 2. Movement of Heterodera glycines second-stage juveniles over time after treatment with abamectin. The percent movement of each treatment population was calculated every hour for $24 \mathrm{~h}$ through sequential digital imaging on a high-resolution flatbed scanner. Data include two experimental runs and six replications per run. 
of nematode populations treated with log-scale dilutions of abamectin. As the concentration of abamectin increased, the percent movement of the nematode populations from each treatment decreased (Fig. 2). The two highest concentrations of abamectin $(10$ and $100 \mu \mathrm{g} / \mathrm{ml})$ inhibited percent movement of $H$. glycines $\mathrm{J} 2$ to levels similar to that of the heat-killed, negative control. Moderate decreases in movement also were observed at the intermediate abamectin concentration of $1 \mu \mathrm{g} / \mathrm{ml}$. These results indicate that high concentrations of abamectin completely inhibit nematode movement, which leads to nematode death. However, intermediate concentrations of abamectin may have sublethal effects, by slowing down movement without killing the nematode. Sublethal effects can be difficult to quantify by manual techniques but can be computed by computer-based tracking techniques.

In the movement analysis method, the population of nematodes was observed only once every hour and not continuously. To track individual nematodes, each worm must be uniquely tagged and the tag must be identifiable in each observation image. There is no way to track individual nematodes over the course of an experiment using our method. There are limitations to comparing populations of nematodes, not individuals, using our method. A nematode that moves but returns to the same position as it was in the previous observation would be considered "not moving" by the program. Similarly, if two nematodes switched locations from one observation to the next, both would be considered "not moving" by the program. Although the likelihood of either of these two possibilities happening with significant frequency seems low, such occurrences cannot be ruled out.

During the first few hours of the experiment, increased movement was observed from nematode populations in all treatments. After a few hours, the movement of the nematodes gradually decreased and remained relatively consistent for the remainder of the observation period. Given that even the heat-killed control exhibited increased movement in the first few hours of analysis, this movement is most likely due to the settling of the nematodes in the semisolid medium, $11.5 \%$ Pluronic gel, used in the experiment. Using a concentration of Pluronic gel lower than the common 23\% (Sasaki-Crawley et al. 2012) allowed for decreased opacity of the medium and increased
A

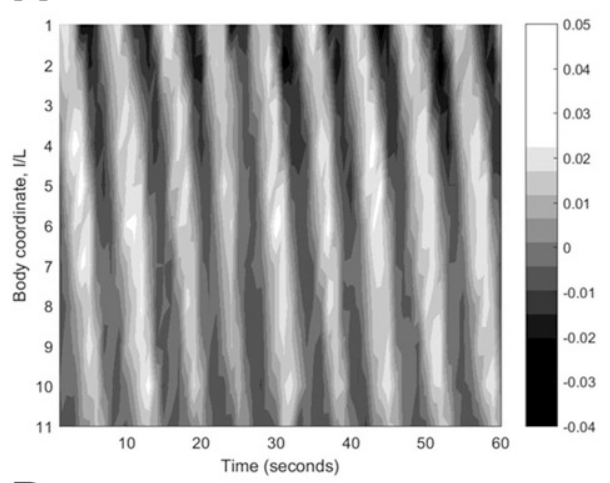

D

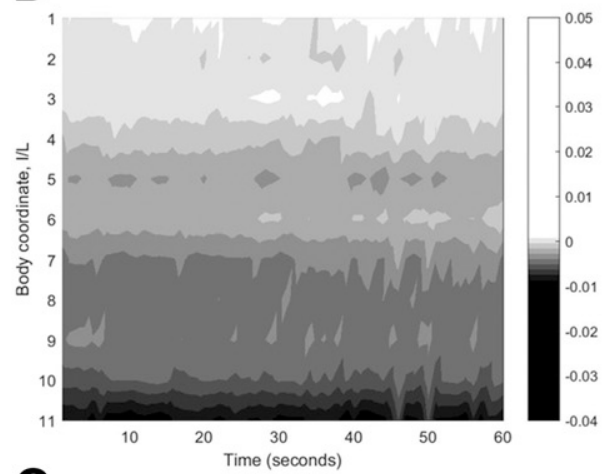

G

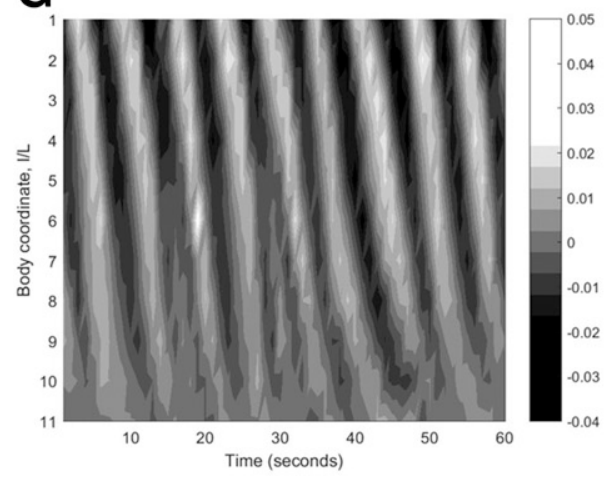

B

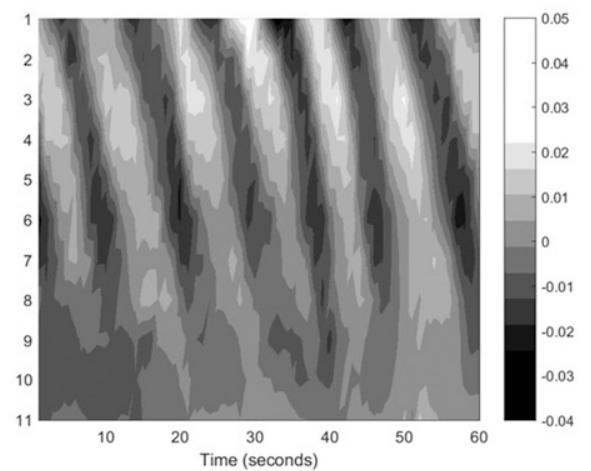

$\mathbf{E}$

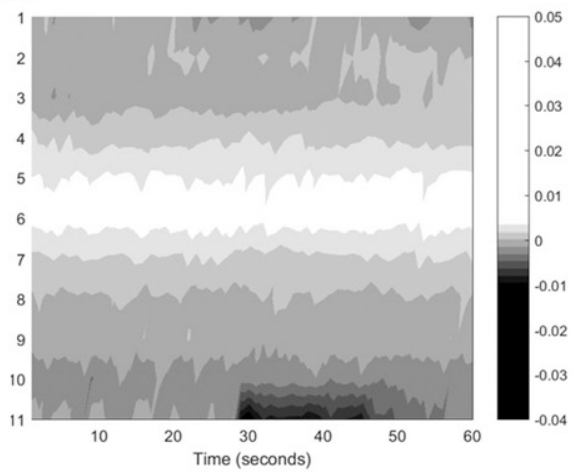

C

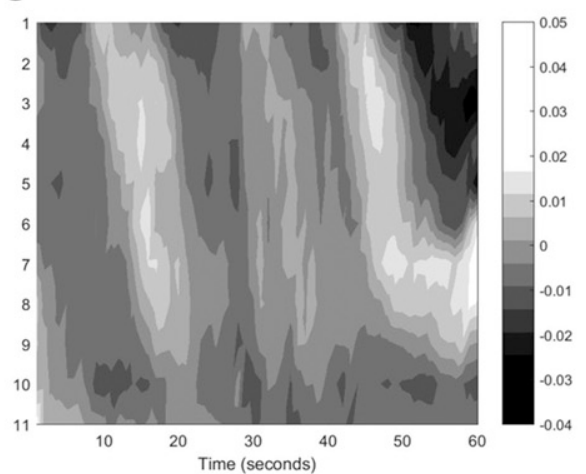

$\mathbf{F}$

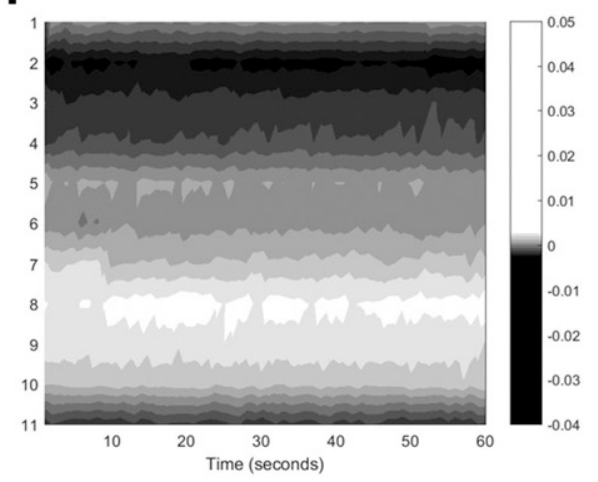

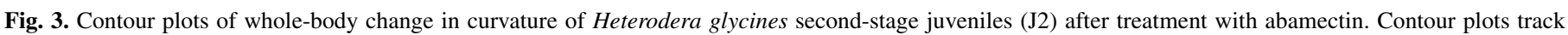

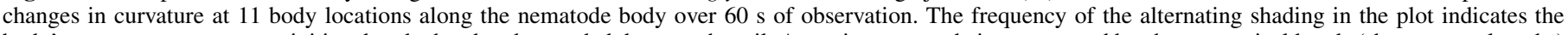

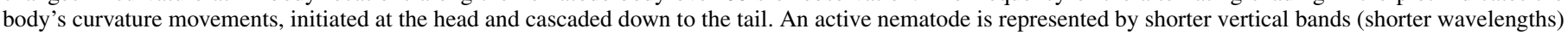

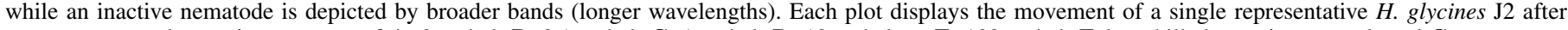
exposure to an abamectin treatment of $\mathbf{A}, 0 \mu \mathrm{g} / \mathrm{ml} ; \mathbf{B}, 0.1 \mu \mathrm{g} / \mathrm{ml} ; \mathbf{C}, 1 \mu \mathrm{g} / \mathrm{ml} ; \mathbf{D}, 10 \mu \mathrm{g} / \mathrm{ml}$; or $\mathbf{E}, 100 \mu \mathrm{g} / \mathrm{ml}$; $\mathbf{F}$, heat-killed negative control; and $\mathbf{G}$, water. 
visibility of nematodes in the scanned images. However, the semisolid nature of the more dilute medium allowed the nematodes to sink down from the surface until they settled on the bottom of the wells.

Motion analysis of individual nematodes measured microscopic changes in speed and in curvature of $H$. glycines $\mathrm{J} 2$. The sensitivity of this technique was determined by measuring differences in nematode motion at 13 different body locations every $0.1 \mathrm{~s}$ following exposure to varying concentrations of abamectin. Plotting whole-body changes in curvature through contour plots (Fig. 3A to $G$ ) allowed for visualization of changes in nematode motion over time. Motion, when measured by change in curvature, cascades across the nematode body starting in the anterior end (or head) and progressing toward the posterior end (or tail) (Lebois et al. 2012). Treatments with lower abamectin concentrations $(0$ and $0.1 \mu \mathrm{g} / \mathrm{ml})$ and the water control showed waves of changes in curvature moving across the body over time, as represented by thin vertical bands in the contour plots. In contrast, nematodes exposed to higher concentrations of abamectin (10 and $100 \mu \mathrm{g} / \mathrm{ml}$ ), and the heat-killed negative control, showed no changes in curvature over time, as shown by the lack of vertical bands.

As the concentration of abamectin increased, there was a significant decrease in both the average speed (Fig. 4) and average change in curvature (Fig. 5) of the nematodes in the experiments at both 0 and $24 \mathrm{~h}$. The changes in nematode behavior, when analyzed for average speed, corresponded to similar changes in curvature. Observations of motion at different tracking points along the nematode body enabled comparison of the response of different body areas to a given treatment. Greater changes in motion (both speed and change in curvature) were observed at the anterior end (or head) compared with posterior end (or tail) of the nematodes for all treatments. Speed and curvature measurements were taken at 0 and $24 \mathrm{~h}$ to determine whether the effects of abamectin diminish over time. On average, there was a $6 \%$ decrease in both speed and change in curvature after $24 \mathrm{~h}$ across all treatments. This overall decrease in motion over time possibly can be attributed to a variety of factors such as a decrease in nematode viability after experimental preparation and handling.

Speed measurements are strong indications of nematode movement but can be influenced by outside disruptions to the experimental setup such as table vibrations affecting the microscope or camera stability or changes in the volume of water in the microscope slide. Curvature measurements were additional and complementary to speed measurements in the motion analysis assay. Calculating the change in curvature in conjunction with speed helped determine whether any outside influences caused changes in nematode movement. Because nematode movement is the result of contracting and relaxing of dorsal and ventral muscles (Perry and Curtis 2013), changes in curvature must be present for true movement to occur. If changes in speed occur without corresponding changes in curvature, then movement most likely was due to outside influences; whereas, if changes in speed are accompanied by changes in curvature, then the observations of motion were likely true movement of the nematode.
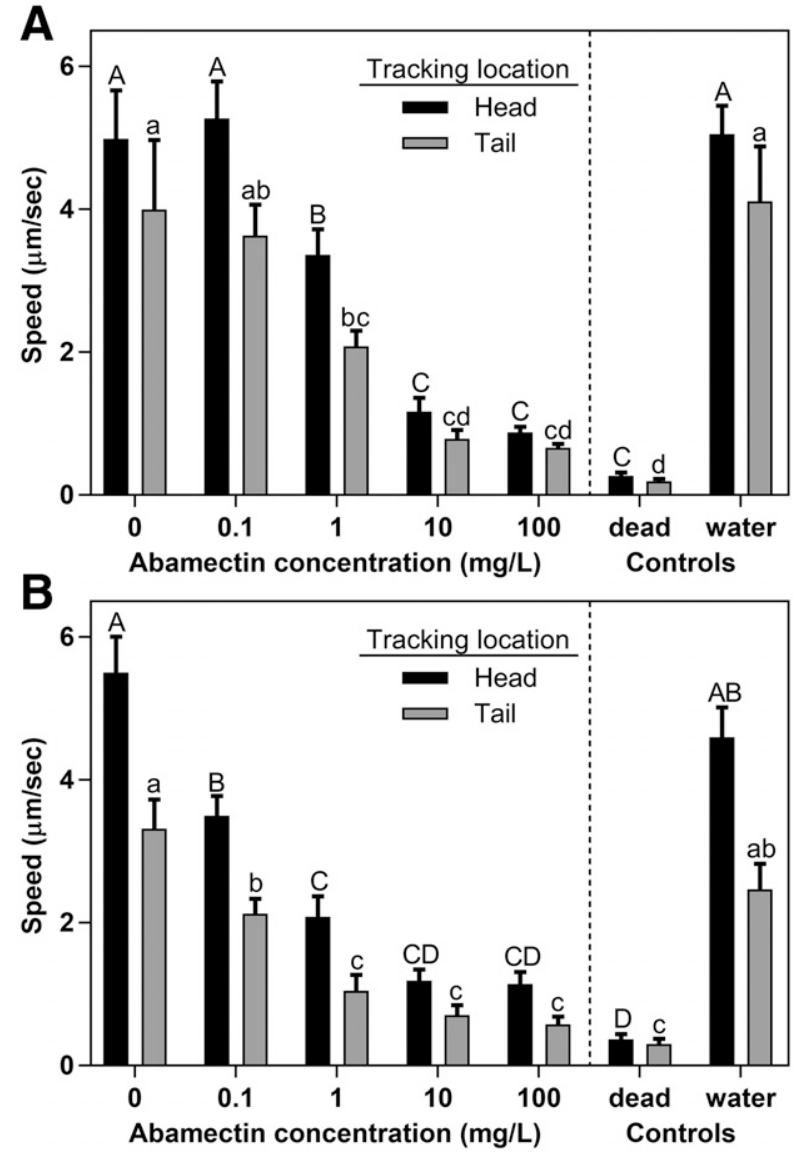

Fig. 4. Speed of motion of Heterodera glycines second-stage juveniles after treatment with various abamectin concentrations. Bars represent average speeds (micrometers per second) of the anterior end (or head; black bar) and the posterior end (or tail; gray bar) of the nematodes over $60 \mathrm{~s}$ of observation at $\mathbf{A}, 0 \mathrm{~h}$ and $\mathbf{B}, 24 \mathrm{~h}$ after incubation. The speed of motion was analyzed separately for the head (uppercase letters) and tail (lowercase letters). Uppercase letters above the black bars and lowercase letters above the gray bars indicate differences in speed according to Tukey's honestly significant difference test $(\alpha=0.05)$.
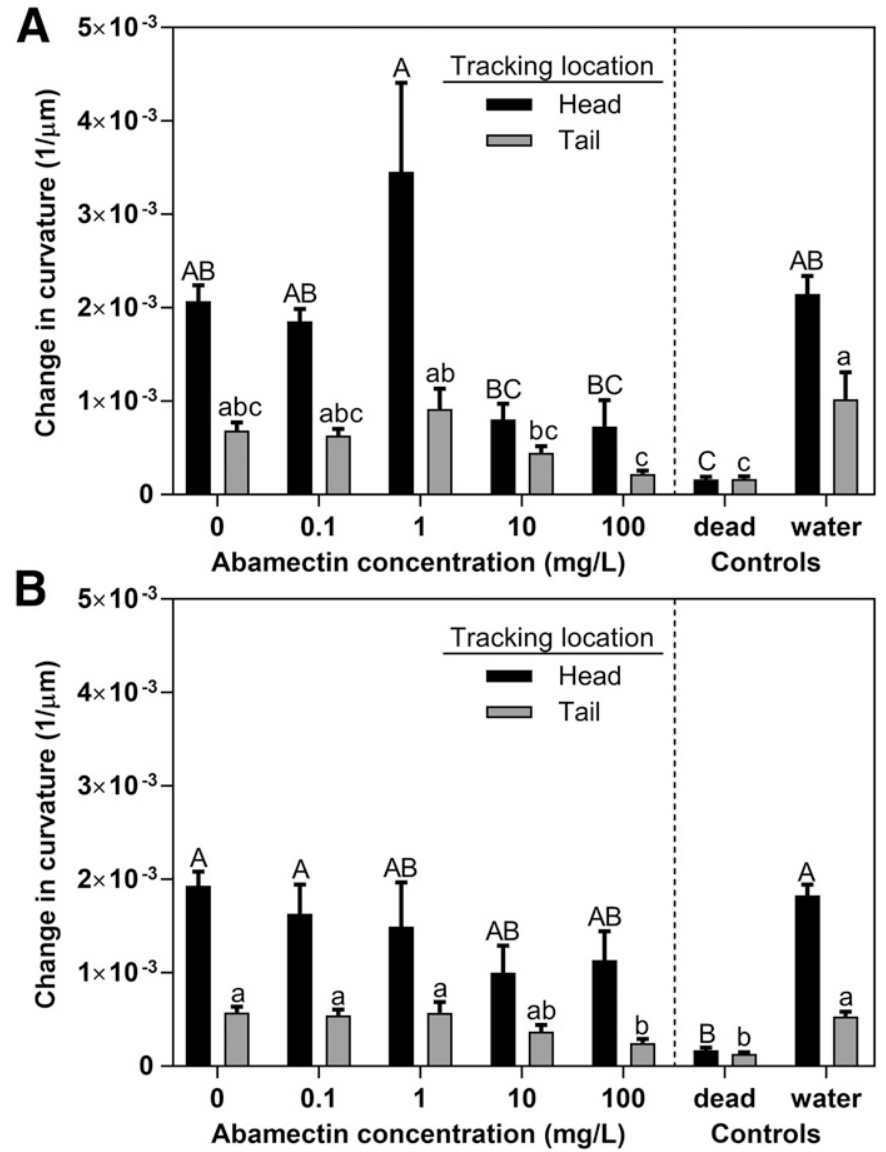

Fig. 5. Change in curvature of motion of Heterodera glycines second-stage juveniles after treatment with abamectin. Bars represent average changes in curvature $(1 / \mu \mathrm{m})$ over $60 \mathrm{~s}$ of observation at $\mathbf{A}, 0 \mathrm{~h}$ and $\mathbf{B}, 24 \mathrm{~h}$ after incubation. Black bars represent the anterior tracking point (head) and gray bars represent the posterior tracking point (tail). The change in curvature was analyzed separately for the head (uppercase letters) and tail (lowercase letters). Uppercase letters above the black bars and lowercase letters above the gray bars indicate differences in change in curvature according to Tukey's honestly significant difference test $(\alpha=0.05)$. 
If an experiment similar to ours was conducted with a range of abamectin concentrations using manual observation methods, the task would be immensely time consuming and only a fraction of the number of experimental observations and replications could be conducted within the same time frame. Automating this process with the high-resolution scanner and custom software increased the productivity potential of such experiments while also adding a level of quantitative data that could not be previously estimated with manual observation methods. The ability to analyze the movement of a population of nematodes in the well of a tissue culture plate through collections of images hourly but allowing for analysis of the images at the convenience of the researcher makes this an easy-touse and time-saving method. Observations of population movement over a 24-h period, rather than a single observation, also allows the researcher to detect the duration of effects of a nematicide, to determine whether there are any quenching effects of the treatment or time-delayed responses. With the aid of computer software, motion analysis of individual nematodes measured and quantified microscopic changes in nematode positioning that likely would otherwise go unnoticed to average human observation. Individual motion analysis has the sensitivity to measure minute changes in nematode movement such as subtle twitches, enabling the researcher to measure the effects of the target nematicide and determine whether any sublethal effects exist. This is especially important when studying nematodes such as $H$. glycines, which may enter into a semi-immobile state (Schroeder and MacGuidwin 2010), where they may exhibit minimal movement. In the experiments described herein, abamectin had an immediate and lasting effect on $H$. glycines $\mathrm{J} 2$ at higher concentrations (10 and $100 \mu \mathrm{g} / \mathrm{ml}$ ) without recovery of nematode mobility.

At first, the data obtained from experiments with the range of abamectin concentrations using both methods were analyzed with abamectin concentration considered a continuous variable and were fitted to logistic curves. However, such analyses had low $R^{2}$ values for the fitted logistic curves (data not shown). Alternatively, the treatments were treated as discrete, categorical variables. Because the treatments were treated as categorical, an effective concentration or inhibitory concentration of abamectin could not be calculated. Consequently, this study refers to the optimal concentration of abamectin for nematicidal activity as an effective range.

Both methods described herein provided quantitative data of nematode behavior but on different scales. Each analysis can be performed independent of the other but results from both methods were complementary. Movement analysis of nematode populations provided a higher-throughput technique to screen multiple treatments and test multiple replications simultaneously. Measuring nematode movement on the population scale allowed for the observation of large numbers of nematodes in a small amount of time and space. This method would be ideal for screening the efficacy of potential nematicides in a research and development setting. Once a candidate nematicide has been identified, motion analysis of individual nematodes would provide information on how the nematicide affected specific aspects of the behavior of the nematode on a singleorganism level. Results from motion analysis of individual nematodes can then be used to determine the minimum concentration required of the candidate nematicide for lethal or sublethal effects by comparing measurements of speed and change in curvature to controls.

There have been several studies on the effectiveness of abamectin on various plant-parasitic nematodes such as $H$. schachtii, Meloidogyne incognita, Pratylenchus zeae, and Rotylenchulus reniformis (Cabrera et al. 2009; Faske and Starr 2006) but no reports about in vitro effects of abamectin on $H$. glycines. Using abamectin in both assays described herein not only demonstrated the specificity of each analysis but also provided the first in vitro sensitivity data of $H$. glycines to abamectin. This study demonstrated the effective lethal range of abamectin (i.e., inhibition of movement and motion), with irreversible effects, between 1 and $10 \mu \mathrm{g} / \mathrm{ml}$, and sublethal effects at concentrations as low as $0.1 \mu \mathrm{g} / \mathrm{ml}$. The in vitro lethal range of abamectin on $H$. glycines obtained here was similar to lethal concentrations reported for $M$. incognita at $1.56 \mu \mathrm{g} / \mathrm{ml}$ (Faske and Starr 2006) but far different from the $32.9 \mu \mathrm{g} / \mathrm{ml}$ reported for $R$. reniformis (Faske and Starr 2006).

These methods may be viable tools for nematicide research and assist in the development of new products for the emerging nematodeprotectant seed treatment market. Future work with these methods need not be limited to nematicides but can incorporate other microorganisms, including biological control candidates, root leachates, other nematodes, and soil extracts, to develop a better understanding of how the soil environment plays a role in nematode movement.

\section{LITERATURE CITED}

Allen, T., Bradley, C., Sisson, A., Byamukama, E., Chilvers, M., Coker, C., Collins, A., Damicone, J., Dorrance, A., Dufault, N., Esker, P., Faske, T., Giesler, L., Grybauskas, A., Hershman, D., Hollier, C., Isakeit, T., Jardine, D., Kelly, H., Kemerait, R., Kleczewski, N., Koenning, S., Kurle, J., Malvick, D., Markell, S., Mehl, H., Mueller, D., Mueller, J., Mulrooney, R., Nelson, B., Newman, M., Osborne, L., Overstreet, C., Padgett, G., Phipps, P., Price, P., Sikora, E., Smith, D., Spurlock, T., Tande, C., Tenuta, A., Wise, K., and Wrather, J. 2017. Soybean yield loss estimates due to diseases in the United States and Ontario, Canada, from 2010 to 2014. Plant Health Prog. 18:19-21.

Beeman, A. Q., Njus, Z., Pandey, S., and Tylka, G. L. 2016. Chip technologies for screening chemical and biological agents against plant-parasitic nematodes. Phytopathology 106:1563-1571.

Cabrera, J. A., Kiewnick, S., Grimm, C., Dababat, A. A., and Sikora, R. A. 2009. Efficacy of abamectin seed treatment on Pratylenchus zeae, Meloidogyne incognita and Heterodera schachtii. J. Plant Dis. Prot. 116:124-128.

Carins, E. J., Sasser, J. N., and Jenkins, W. R. 1960. Pages 33-84 in: Methods in Nematology. Nematology, Fundamentals and Recent Advances with Emphasis on Plant Parasitic and Soil Forms. University of North Carolina Press, Chapel Hill.

Cayrol, J. C., Castet, R., and Samson, R. A. 1986. Comparative activity of different Hirsutella species towards three plant parasitic nematodes. Rev. Nematol. 9:412-414.

Chen, S. Y., and Dickson, D. W. 2000. A technique for determining live second-stage juveniles of Heterodera glycines. J. Nematol. 32:117-121.

Dusenbery, D. B. 1985. Using a microcomputer and video camera to simultaneously track 25 animals. Comput. Biol. Med. 15:169-175.

Faghihi, J., and Ferris, J. M. 2000. An efficient new device to release eggs from Heterodera glycines. J. Nematol. 32:411-413.

Faske, T. R., and Starr, J. L. 2006. Sensitivity of Meloidogyne incognita and Rotylenchulus reniformis to abamectin. J. Nematol. 38:240-244.

Hooper, D. J. 1986. Pages 59-80 in: Handling, Fixing, Staining, and Mounting Nematodes. Laboratory Methods for Work with Plant and Soil Nematodes. Her Majesty's Stationary Office, London.

Husson, S. J., Costa, W. S., Schmitt, C., and Gottschalk, A. 2005. Keeping track of worm trackers. In: WormBook. O. Hobert, ed. The C. elegans Research Community, Pasadena, CA.

Jenkins, W. 1964. A rapid centrifugal-flotation technique for separating nematodes from soil. Plant Dis. Rep. 48:692.

Kass, I. S., Stretton, A. O. W., and Wang, C. C. 1984. The effects of avermectin and drugs related to acetylcholine and 4-aminobutyric acid on neurotransmission in Ascaris suum. Mol. Biochem. Parasitol. 13:213-225.

Koenning, S. R., and Wrather, J. A. 2010. Suppression of soybean yield potential in the continental United States by plant diseases from 2006 to 2009. Online publication. Plant Health Prog. doi.org/10.1094/PHP-2010-1122-01-RS

Korta, J., Clark, D. A., Gabel, C. V., Mahadevan, L., and Samuel, A. D. T. 2007. Mechanosensation and mechanical load modulate the locomotry gait of swimming C. elegans. J. Exp. Biol. 210:2383-2389.

Lebois, F., Sauvage, P., Py, C., Cardoso, O., Ladoux, B., Hersen, P., and Meglio, J. M. 2012. Locomotion control of Caenorhabditis elegans through confinement. Biophys. J. 102:2791-2798.

Munkvold, G. P., Watrin, C., Scheller, M., Zeun, R., and Olaya, G. 2014. Benefits of chemical seed treatments on crop yield and quality. Pages 89-103 in: Global Perspectives on the Health of Seeds and Plant Propagation Material. M. L. Gullino and G. Munkvold, eds. Springer, Dordrecht, The Netherlands.

Njus, Z., Feldman, D., Brien, R., Kong, T., Kalwa, U., and Pandey, S. 2015. Characterizing the effect of static magnetic fields on C. elegans using microfluidics. Adv. Biosci. Biotechnol. 6:583-591.

Njus, Z. 2017. Behavioral assays with smart worm recognition programs applied to plant parasitic and human parasitic nematodes. Iowa State University Graduate Theses and Dissertations. Number 15986. https://lib.dr. iastate.edu/etd/15986

Ogiga, I. R., and Estey, R. H. 1974. The use of Meldola blue and Nile blue a, for distinguishing dead from living nematodes. Nematologica 20:271-276. 
Peet, F. G., Panesar, T. S., Sahota, T. S., and Sutherland, J. R. 1990. A digital image analysis system for comparing groups of small nematodes. J. Nematol. 22:407-413.

Perry, R. N., and Curtis, R. H. C. 2013. Behaviour and sensory perception. Pages 246-273 in: Plant Nematology, 2nd ed. R. N. Perry and M. Moens, eds. CABI, Oxfordshire, UK.

Sasaki-Crawley, A., Curtis, R., Birkett, M., Papadopoulos, A., Blackshar, R., and Pickett, J. 2012. The use of Pluronic F-127 to study the development of the potato cyst nematode, Globodera pallida. Nematology 14:869-873.

Schroeder, N. E., and MacGuidwin, A. E. 2010. Behavioral quiescence reduces the penetration and toxicity of exogenous compounds in second-stage juveniles of Heterodera glycines. Nematology 12:277-287.
Wong, A. T. S., Tylka, G. L., and Hartzler, R. G. 1993. Effects of eight herbicides on in vitro hatching of Heterodera glycines. J. Nematol. 25:578-584.

Wright, D. J., Birtle, A. J., Corps, A. E., and Dybas, R. A. 1983. Efficacy of avermectins against a plant parasitic nematode. Ann. Appl. Biol. 103: 465-470.

Wright, D. J., Birtle, A. J., and Roberts, I. T. J. 1984. Triphasic locomotor response of a plant-parasitic nematode to avermectin: Inhibition by the GABA antagonists bicuculline and picrotoxin. Parasitology 88:375-382.

Xiang, N., and Lawrence, K. S. 2016. Optimization of in vitro techniques for distinguishing between live and dead second stage juveniles of Heterodera glycines and Meloidogyne incognita. PLoS One 11:e0154818. 